\title{
LEVANTAMENTO FLORÍSTICO DE FLORESTA ATLÂNTICA NO SUL DO ESTADO DE SÃO PAULO, PARQUE ESTADUAL INTERVALES, BASE SAIBADELA
}

\author{
Valesca Bononi Zipparro ${ }^{1,3}$, Frederico Augusto G. Guilherme ${ }^{1}$, Renata J. Almeida-Scabbia ${ }^{2}$ \& L. Patrícia C. \\ Morellato $^{1}$
}

Biota Neotropica v5 (n1) -http://www.biotaneotropica.org.br/v5n1/pt/abstract?inventory+BN02605012005

Recebido: 01/10/2004

Revisado: $13 / 12 / 2004$

Publicado: 01/01/2005

\begin{abstract}
${ }^{1}$ Universidade Estadual Paulista, Instituto de Biociências, Departamento de Botânica, Grupo de Fenologia e Dispersão de Sementes, Caixa Postal 199, 13506-900 Rio Claro, SP, Brasil.

${ }^{2}$ Endereço atual: Fundação Instituto de Ensino para Osasco - FIEO, Av. Franz Voegeli, 300, 06020-190, Osasco, SP

${ }^{3}$ Autor para correspondência: zipparro@rc.unesp.br
\end{abstract}

\begin{abstract}
A floristic survey was carried out in the Parque Estadual Intervales (PEI), Base Saibadela (24¹4’08"S, 4804’42"W), São Paulo State, southeastern Brazil. The PEI reserve, along with other conservation unities, compose the largest preserved area of Atlantic rain forest remained today. Monthly collections of botanical material were conducted from 1993 to 1997 and 1999 to 2002, mainly along trails and plots inside the forest. A total of 436 plant species, 74 monocots and 362 eudicotiledons (here including the parafiletic groups) were sampled, including five new species. The richness of the flora at Base Saibadela was similar to that observed for other Atlantic forest sites. The trees represented $57.1 \%$ of the species, followed by epiphytes (12.6\%), lianas (10.3\%), herbs (9.4\%) and shrubs (9.2\%). The families Myrtaceae (55), Rubiaceae (32), Fabaceae (25), Melastomataceae (23), Araceae (20), Lauraceae Orchidaceae and Solanaceae (14 species each one) showed the highest number of species. The genus Eugenia with 25 species was richest genera surveyed. Seed dispersal by animals was expressively higher than other modes, which comprises $80.7 \%$ of the 419 species where seed dispersal mode was determined, confirming the pattern found for other tropical wet forests, and highlighting the importance of plant-animal interactions to the Atlantic forest.
\end{abstract}

Key words: Biodiversity, tropical rainforest, Atlantic forest, floristic inventory, dispersion syndromes.

\section{Resumo}

Foi realizado um levantamento florístico no Parque Estadual Intervales, Base Saibadela (24¹4’08"S, 4804’42"W), que faz parte da maior área contínua de Floresta Atlântica brasileira remanescente, situada no sul do Estado de São Paulo. Durante os anos de 1993-1997 e 1999-2002 foram feitas coletas mensais de material botânico, principalmente ao longo de trilhas e parcelas no interior da floresta. Foi registrado um total de 436 espécies vegetais, 74 monocotiledôneas (incluindo os grupos parafiléticos), sendo descobertas cinco espécies novas. A riqueza florística na Base Saibadela foi compatível com a riqueza florística encontrada em outras localidades de Floresta Atlântica. O hábito arbóreo representou 57,1\% das espécies, seguido de epífitas (12,6\%), lianas (10,3\%), herbáceas (9,4\%) e arbustos (9,2\%). As famílias Myrtaceae (55), Rubiaceae (32), Fabaceae (25), Melastomataceae (23), Araceae (20), Lauraceae, Orchidaceae e Solanaceae (14 espécies cada) apresentaram a maior riqueza florística. O gênero Eugenia apresentou 25 espécies, destacando-se dos demais gêneros encontrados. A dispersão por animais foi expressivamente maior do que os demais modos, representando 80,7\% das 419 espécies cuja síndrome foi determinada, confirmando o padrão encontrado para outras florestas tropicais úmidas e ressaltando a importância da fauna na Floresta Atlântica.

Palavras-chave: Biodiversidade, floresta tropical, floresta atlântica, inventário florístico, modos de dispersão 


\section{Introdução}

A variedade de clima e relevo do Domínio atlântico (sensu Ab’ Saber 1977), num sentido amplo, proporciona uma grande diversidade de ambientes e ecossistemas complexos a ele associados, englobando a floresta pluvial atlântica, a floresta estacional semidecidual, mangues, restingas e campos de altitude (Mantovani 1990, LeitãoFilho 1994, Mantovani 1998, Ivanauskas et al. 2000, OliveiraFilho \& Fontes 2000, Scudeller et al. 2001, Scarano 2002). No Estado de São Paulo, essa variação ambiental é abrupta, pois a vegetação da Serra do Mar, sob influência mais direta do oceano, recebe em torno de $3.600 \mathrm{~mm} / \mathrm{ano}$ de chuva, enquanto a maiores distâncias do oceano o clima se torna sazonal, com chuvas entre 1.300 e 1.600 mm/ano (OliveiraFilho \& Fontes 2000). Ao longo dessas zonas climáticas observa-se uma diferenciação das formações florestais, com a floresta estacional semidecidual no interior do Estado e a floresta pluvial atlântica ou floresta atlântica propriamente dita ao longo da costa, recobrindo a Serra do Mar (OliveiraFilho \& Fontes 2000).

Devido ao acelerado processo de devastação, graças à intensa atividade agropastoril, industrial e urbana, a floresta atlântica foi praticamente dizimada ao longo dos séculos após o descobrimento, restando hoje entre 5-8\% da cobertura original (Mori 1988, Dean 1995, Morellato \& Haddad 2000). Considerada como um dos maiores centros de biodiversidade, com altos níveis de endemismo, a floresta atlântica está entre as oito áreas prioritárias ('hotspots') do planeta, em termos de estratégias de conservação (Myers et al. 2000). Contudo, os remanescentes estão sujeitos a intervenções e ameaças constantes, pois se encontram próximos dos grandes centros urbanos brasileiros ou estão envolvidos por vastas plantações de café, cana-de-açúcar e eucalipto (Dean 1995, Morellato \& Haddad 2000).

Boa parte dos remanescentes de Floresta Atlântica mais expressivos e extensos estão situados no estado de São Paulo, graças ao relevo acidentado da Serra do Mar e da Serra de Paranapiacaba (Leitão Filho 1994), com 40\% concentrados na região do Vale do Rio Ribeira do Iguape, sul do estado (Silva Matos \& Bovi 2002). Em razão de algumas localidades apresentarem relevo e solos propícios para agricultura, essa região tem expandido sua fronteira agrícola e causado sérios problemas para a flora local (Ivanauskas et al. 2001). Essa região e seus arredores é a mais pobre do estado e tem como principais fontes de renda agrícola o cultivo de chá e banana. A exploração do palmitojuçara (Euterpe edulis) também é uma importante fonte de renda e ocorre, principalmente, de forma clandestina, embora ainda existam algumas das poucas populações intactas de palmito ao longo de toda a Floresta Atlântica brasileira (Galetti \& Chivers 1995). Nesta região insere-se a Base Saibadela, pertencente ao Parque Estadual Intervales, uma Unidade de Conservação de elevada importância ecológica que, juntamente com outras Unidades de Conservação, formam a maior área contínua de floresta atlântica brasileira, abrigando uma biodiversidade ainda pouco conhecida (Fundação Florestal 2001).

Este estudo tem como objetivos incrementar as informações sobre a diversidade e distribuição de espécies vegetais na Floresta Atlântica, apresentando um levantamento florístico no Parque Estadual Intervales e a classificação das espécies quanto às formas de vida e modos de dispersão.

\section{Material e Métodos}

\section{1 Área de estudo}

O estudo foi realizado no Parque Estadual Intervales

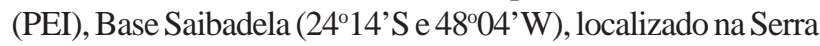
de Paranapiacaba, município de Sete Barras, sul do Estado de São Paulo (Figura 1). O PEI possui uma área de aproximadamente 48.000 ha e é circundado por outras três Unidades de Conservação (Parque Estadual Carlos Botelho, Estação Ecológica do Xitué e Parque Estadual Turístico do Alto Ribeira), totalizando cerca de 200.000 ha (Fundação Florestal 2001). Aárea é coberta, principalmente, por floresta atlântica primária com pouca intervenção antrópica (Figura 2A) e situa-se nos domínios da Floresta Ombrófila Densa Submontana (IBGE 1991).

O PEI tem relevo bastante acidentado e encontra-se em altitudes que variam de 60 a 1.100 m; na Base Saibadela, a altitude varia em torno de 70 a 250 m (Fundação Florestal 2001). Em termos geomorfológicos, a Base Saibadela localizase na Serrania Costeira da Serra de Paranapiacaba, a qual possui amplas feições erosivas de aspecto montanhoso, formadas por escarpas íngremes e vales profundos, que representam o rebordo do Planalto Cristalino Atlântico, constituídos principalmente por rochas graníticas e gnáissicas de elevada complexidade estrutural (Almeida 1974). Predominam no local solos distróficos com poucos nutrientes e acidez elevada, alta saturação por Al trocável e baixa saturação por bases, proporcionadas pelos baixos teores de Ca, Mg e K trocáveis (Guilherme et al. 2004).

Os dados climáticos registrados na própria área de estudo (1994 a 1996; 2000) apontam para um clima tropical superúmido, sem períodos de déficit hídrico e com chuvas o ano todo, com precipitação média anual de $4.000 \mathrm{~mm}$ (Morellato et al. 2000). Há uma estação mais fria e menos chuvosa de abril a agosto, com temperatura média do mês mais frio (julho) de $18^{\circ} \mathrm{C}$ e precipitação acima de $100 \mathrm{~mm}$ e a outra estação, mais quente e chuvosa, de setembro a março, com temperatura média do mês mais quente (janeiro) de 28,1 ${ }^{\circ} \mathrm{C}$ e precipitação acima de $250 \mathrm{~mm}$ (Morellato et al. 2000). Segundo Setzer (1966), a região apresenta clima do tipo Cfa no sistema de Köeppen, quente sem estação seca. 


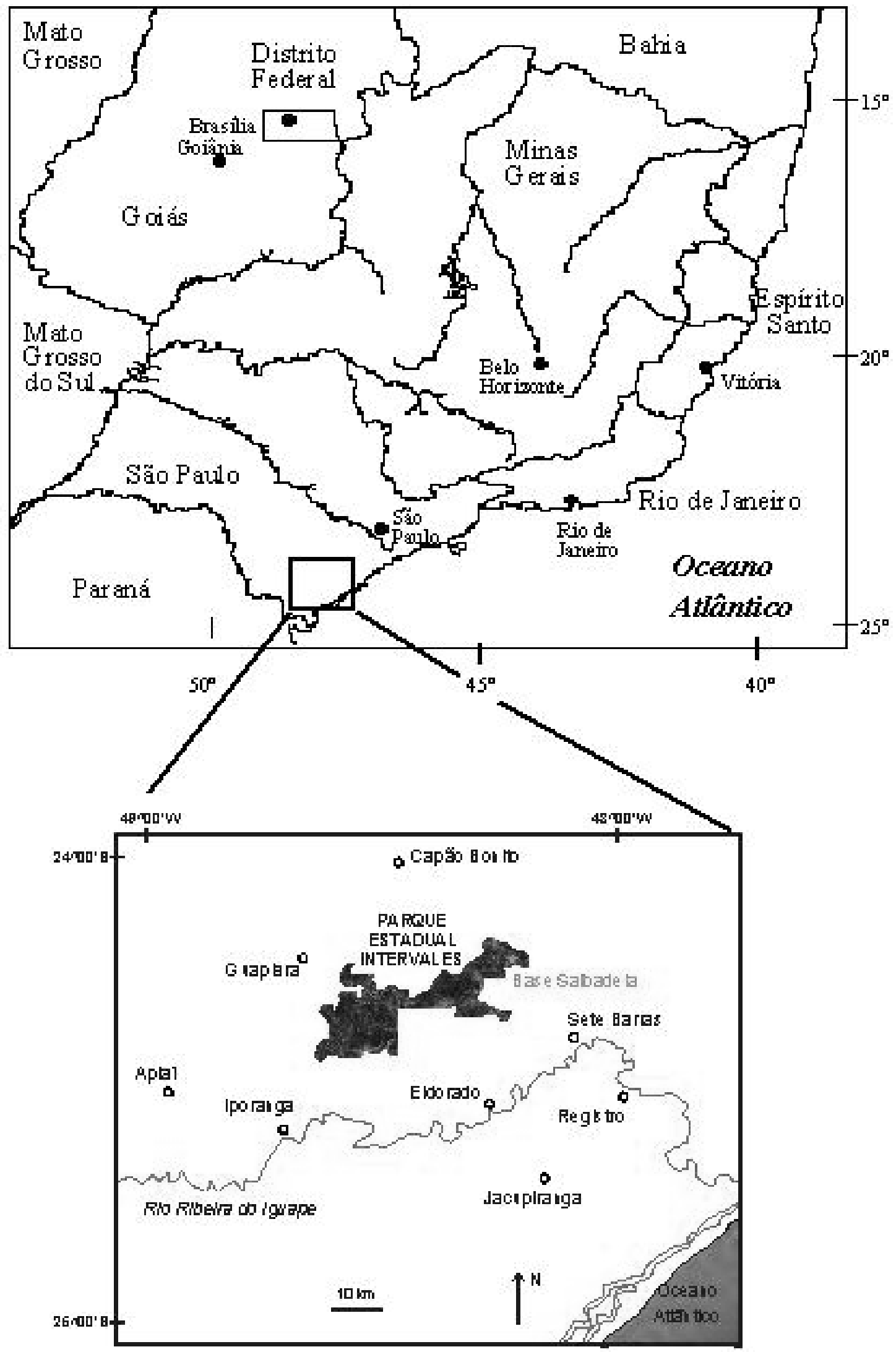

Figura 1: Localização do Parque Estadual Intervales e da Base Saibadela, município de Sete Barras, SP. 


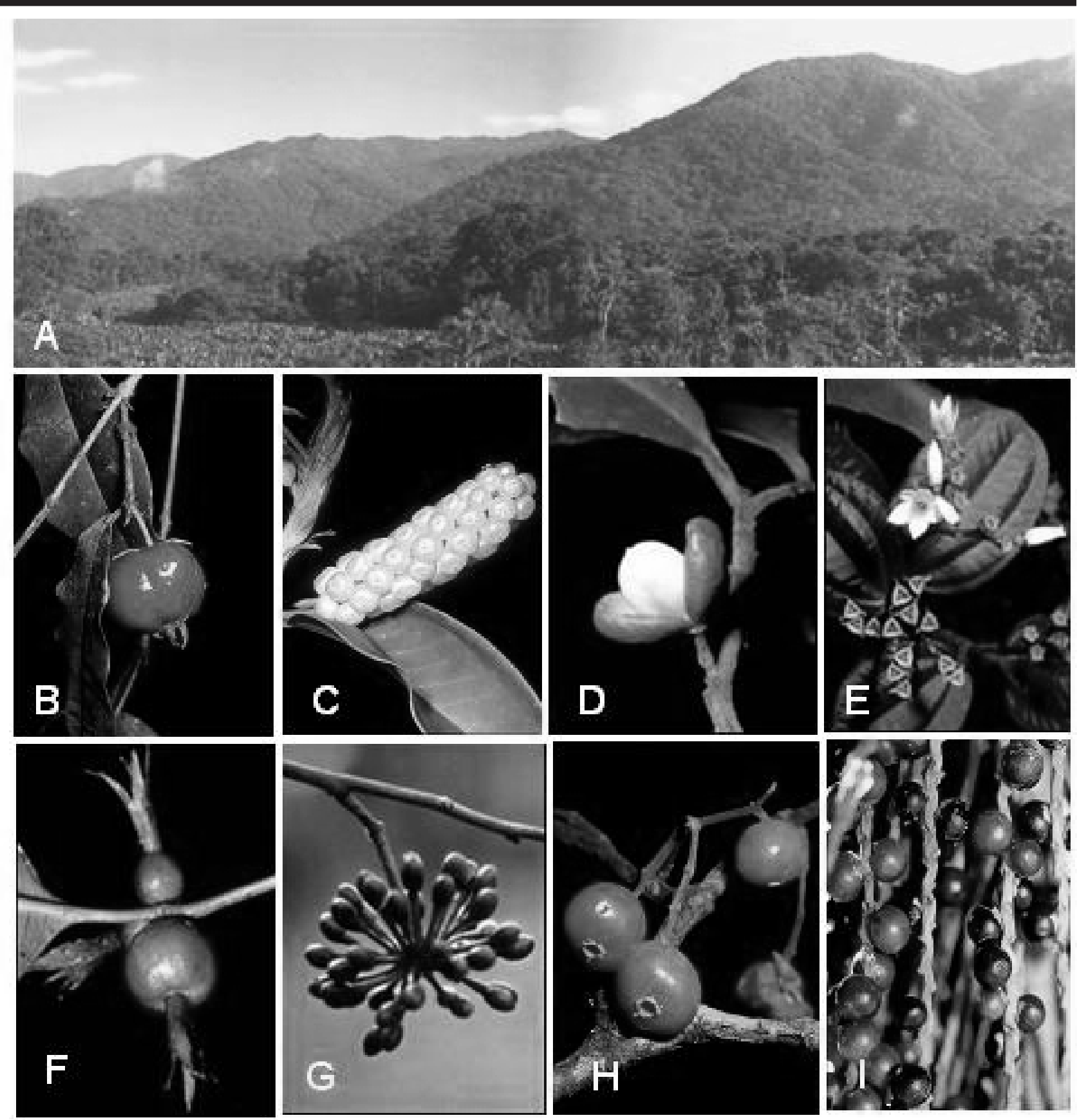

Figura 2: A. Aspecto geral da área estudada, Parque Estadual Intervales, Sete Barras, SP. B. Eugenia cuprea (O. Berg.) Nied. (murtinha - Myrtaceae); C. Anthurium scandens (Aubl.) Engl. (Araceae); D. Maytenus schumanniana Loes. (Celastraceae); E. Bertolonia mosenii Cogn. (Melastomataceae); F. Psychotria suterella Muell. Arg. (erva-de-anta - Rubiaceae); G. Guatteria australis A. St. Hill. (pindaúva-branca - Annonaceae); H. Plinia complanata M.L.Kawasaki \& B.Holst (Myrtaceae); I. Euterpe edulis Mart (palmito - Arecaceae). Fotos B, C e H por M.A. Pizo; I por M. Galetti. 


\subsection{Levantamento florístico}

As coletas foram feitas mensalmente entre os anos de 1993 e 2002, com exceção de 1997 e 1998, quando a direção do parque decidiu interromper as pesquisas na Base Saibadela, e limitaram-se às angiospermas. O esforço de coleta concentrou-se nos blocos amostrais - cerca de 2 ha. - pertencentes a um estudo fitossociológico (Guilherme et al. 2004) e ao longo de trilhas no interior da floresta (AlmeidaScabbia 1996, Zipparro 2004), sendo ainda realizadas coletas em locais menos acessíveis da floresta. O material botânico em estado fértil foi coletado e herborizado, anotando-se a forma de vida das espécies, classificadas em sete hábitos descritos a seguir: 1. árvore: plantas com fuste lenhoso e, em geral, com diâmetro a altura do meio $\geq 5 \mathrm{~cm}$ e mais de $3 \mathrm{~m}$ de altura; 2. arbusto: plantas com base do caule lenhoso e, em geral, com diâmetro a altura do meio $\leq 5 \mathrm{~cm}$ e com menos de 3 m de altura; 3. liana: plantas lenhosas ou não, com hábito escandente ou trepador; 4. estranguladora: plantas lenhosas com hábito estrangulador; 5: hebácea: ervas terrestres e sem caule lenhoso; 6: epífita: plantas que utilizam outras plantas, em geral lenhosas, como suporte, sem causar injúrias ao hospedeiro; 7: parasita: plantas que vivem sobre outras, recorrendo ao sistema vascular do hospedeiro para suprir suas necessidades nutricionais. Embora não apresentem caule lenhoso (estipe), as espécies da família Arecaceae foram consideradas como árvores ou arbustos, dependendo das características apresentadas para cada espécie. As identificações foram feitas com o auxílio da literatura específica, consultas à especialistas, além de comparações com material dos Herbários SP, UEC e ESA, além do HRCB, onde o material encontra-se depositado. Boa parte dessa coleção botânica também está incorporada no Herbário SP, visando contribuir para o Projeto Flora Fanerogâmica do Estado de São Paulo. As espécies foram agrupadas nas famílias reconhecidas pelo Angiosperm Phylogeny Group (APG 2003).

As informações sobre modos de dispersão foram baseadas em observações de campo e outros estudos dessa natureza realizados no local (Vieira \& Izar 1999, Galetti et al. 2000, Morellato et al. 2000, Pizo \& Oliveira 2000, Pizo 2002, Pizo \& Morellato 2002), e foram agrupadas em quatro modos de dispersão: zoocoria - diásporos adaptados à dispersão por animais; anemocoria - diásporos adaptados à dispersão pelo vento; hidrocoria - diásporos adaptados à dispersão pela água; autocoria - agrupados em espécies barocóricas (dispersão por gravidade) e com dispersão explosiva.

É importante salientar que o componente arbóreo foi melhor amostrado do que outras formas de vida, devido aos estudos fitossociológicos (Almeida-Scabbia 1996, Guilherme et al. 2004) e fenológicos (Morellato et al. 2000, Zipparro 2004) realizados no local.

\section{Resultados e Discussão}

No total, registradas 436 espécies, 74 monocotiledôneas e 362 eudicotiledôneas (incluindo os grupos parafiléticos, conforme APG 2003), pertencentes a 233 gêneros e 90 famílias, 16 de monocotiledôneas e 74 de eudicotiledôneas; 17 espécies permaneceram identificadas apenas em nível de família (Tabela 1). Oito famílias mais ricas em espécies, Myrtaceae (55), Rubiaceae (32), Fabaceae (25), Melastomataceae (23), Araceae (20), Lauraceae, Orchidaceae e Solanaceae (14 espécies cada), compreenderam 45,2\% do total de espécies levantadas, comprovando sua grande importância na flora da floresta atlântica do estado de São Paulo (Ivanauskas et al. 2001, Mamede et al. 2004). O gênero Eugenia (25) apresentou número expressivamente maior de espécies, seguido por Anthurium, Maytenus, Miconia e Psychotria, com nove espécies cada (Figura 2B-F). A constatação dessa maior riqueza para Myrtaceae e para o gênero Eugenia corrobora o padrão que tem sido encontrado para floresta atlântica do estado de São Paulo (Sanches et al. 1999, Ivanauskas et al. 2001, Scudeller et al. 2001) e do Brasil (Oliveira Filho \& Fontes 2000). Segundo Mori et al. (1983) e Peixoto \& Gentry (1990), a família Myrtaceae tem grande importância florística em toda costa brasileira, devido à elevada riqueza de suas espécies lenhosas.

Baseado em informações recentes sobre fitogeografia (Mamede et al. 2004), o Parque Estadual Intervales possui espécies endêmicas e com distribuição restrita à costa leste do Brasil, tais como Guatteria australis (SP, PR) (Annonaceae) (Figura 2G), Calycorectes acutatus (MG, SP), Eugenia bocainensis (SP, PR) (Myrtaceae) e Pouteria psammophila (RJ, SP) (Sapotaceae). Outras espécies são registradas pela segunda vez para o estado de São Paulo, tais como Myrceugenia kleinii (Myrtaceae) e Psychotria birotula (Rubiaceae). Plinia complanata (Figura 2H) e $P$. pauciflora (Myrtaceae) (Kawasaki \& Holst 2002) e uma espécie do gênero Cryptocarya (Lauraceae) (P. L. R. Moraes, com. pess.) são táxons arbóreos novos, além de Neomarica sp. - Iridaceae (L. Capellari Jr., com. pess.) e uma espécie do gênero Calathea - Marantaceae (V. L. R. Uliana, com. pess.), ambas plantas herbáceas ainda não descritas pela ciência, totalizando cinco espécies inéditas descobertas na Base Saibadela.

O hábito arbóreo foi o mais comum entre as formas de vida coletadas (Tabela 2), como constatado por Lima \& Guedes-Bruni (1994) e Ivanauskas et al. (2001). Destacaramse no dossel florestal árvores de grande porte, tais como Alchornea triplinervia, Hyeronima alchorneoides, Pseudopiptadenia warmingii, Pterocarpus rohrii, Sloanea guianensis e Virola bicuhyba. Árvores de pequeno e médio porte estão bem representadas pelas espécies Eugenia mosenii, E. cuprea (Figura 2B), Euterpe edulis (Figura 2I), Garcinia gardneriana, Guapira opposita, Ixora burchelliana, Marlierea tomentosa, Mollinedia schottiana e Psychotria suterella (Figura 2F), como constatado por Guilherme et al. (2004). 
As famílias Melastomataceae, Rubiaceae e Solanaceae foram as mais ricas em espécies do componente arbustivo. Estas famílias estão entre as citadas por Tabarelli \& Mantovani (1999) e por Ivanauskas et al. (2001), em áreas de Floresta Atlântica de encosta no Estado de São Paulo, como as de maior riqueza entre as arvoretas e arbustos. Entre as herbáceas podemos destacar a família Cyperaceae, típica das regiões de topo de morros, onde o estrato arbóreo é mais baixo e a incidência luminosa maior. Nos sopés das encostas e nas planícies menos drenadas, devido à proximidade com cursos d'água, predominam agrupamentos de Aphelandra liboniana (Acanthaceae) e, principalmente, touceiras de Calathea spp. (Marantaceae), plantas herbáceas que se reproduzem vegetativamente, por meio de rizomas, ocupando expressivamente vários trechos do subbosque florestal.

Na Base Saibadela, as famílias com maior riqueza entre as lianas foram Fabaceae e Bignoniaceae, que aparecem também como as famílias mais ricas em espécies de lianas na floresta atlântica do estado de São Paulo (Kim 1996, Ivanauskas et al. 2001). Outra característica marcante na floresta atlântica da Base Saibadela é a grande ocorrência de epífitas, com destaque para as espécies da família Araceae, tanto pelo porte de seus indivíduos, como pela sua abundância. As famílias Araceae (19), Orchidaceae (10), Bromeliaceae (9) e Piperaceae (6) apresentaram o maior número de espécies, totalizando $80 \%$ dos epífitos encontrados. A família Araceae foi bem levantada devido a estudo específico sobre hábito alimentar de mamíferos arbóreos (Vieira \& Izar 1999). Orchidaceae provavelmente não teve a maior riqueza florística porque não foi amostrada de forma tão intensa quanto as aráceas. Como é amplamente conhecido em levantamentos de epífitos vasculares, as orquídeas superam todos os outros grupos de plantas em número de espécies epifíticas nos trópicos e subtrópicos (Gentry \& Dodson 1987, Dittrich et al. 1999, Borgo \& Silva 2003). É importante salientar que as pteridófitas, principalmente epifíticas, também são abundantes na Base Saibadela, com cerca de 120 espécies, e serão objeto de estudo específico (A. Salino \& V.A.O. Dittrich, dados não publicados).

A dispersão por animais foi expressivamente maior do que os demais modos, presente em 80,7\% das 419 espécies cujo modo de dispersão foi determinado (Tabela 3). Esse tipo de dispersão englobou 89,2\% das espécies arbustivo-arbóreas no levantamento e este valor é similar ao observado para espécies arbóreas em outras localidades de Floresta Atlântica (Morellato et al. 2000, Talora \& Morellato 2000). Esses valores mostram a importância da fauna para as espécies vegetais e o alto número de interações interespecíficas (conectância), abordando apenas a dispersão de sementes, existentes na Floresta Atlântica. A dispersão por animais também foi predominante entre as 55 espécies epifíticas levantadas (63,6\%), embora a dispersão pelo vento também seja marcante neste grupo $(36,4 \%)$. Madison (1977) afirma que a anemocoria é vantajosa para plantas que habitam o dossel, assim a dispersão pelo vento entre as epífitas é muito mais freqüente do que nas plantas terrícolas de florestas tropicais chuvosas. Lianas também ocupam, predominantemente, a copa das árvores, posição que favorece a dispersão pelo vento, observada em 34,1\% das espécies desse grupo na Base Saibadela. Entretanto, a maioria das lianas apresentou dispersão por animais (58,5\%), diferindo do observado para lianas de florestas estacionais semideciduais do interior do estado de São Paulo (Morellato \& Leitão Filho 1996). Nestas florestas a proporção de anemocoria é superior a 70\% e apenas 18,7\% das espécies de lianas são dispersas por animais, sendo geralmente trepadeiras herbáceas, do sub-bosque (Morellato \& Leitão Filho 1996). Esta elevada proporção de dispersão por animais em floresta atlântica parece relacionada à elevada pluviosidade do local (4.000 mm), já que em florestas úmidas pouco sazonais a proporção de espécies dispersa por animais, em qualquer hábito, supera a daquelas dispersas pelo vento (L. P. C. Morellato, dados não publicados).

Embora reduzida, a cobertura vegetal da floresta atlântica no sul do estado de São Paulo ainda é expressiva em relação ao restante do país e possui uma biodiversidade marcante. A dificuldade de se obter uma medição direta do grau de resiliência em ecossistemas de grande biodiversidade, como a floresta atlântica, onde conectância e força de interações são praticamente impossíveis de serem estimadas para o ecossistema como um todo (Scarano 2004), torna urgente a preservação e o manejo das áreas remanescentes para a conservação das espécies e a conseqüente manutenção da diversidade genética e biológica, até que um conhecimento adequado seja adquirido.

\section{Agradecimentos}

Somos gratos aos taxonomistas inseridos no Projeto Flora do Estado de São Paulo, em especial à Maria Lúcia Kawasaki (Myrtaceae), Ana Paula Prata (Cyperaceae) e Renata G. Uduluscht (Orchidaceae e lianas) e Fábio de Barros (Orchidaceae) pelo auxílio na identificação do material botânico; ao Marco Antonio de Assis pela leitura crítica do manuscrito; ao Marcos P. M. Aidar e a um assessor anônimo pelas sugestões ao manuscrito, ao Instituto Florestal e Fundação Florestal, pela permissão para estudo e apoio logístico e à FAPESP (proc. $n^{\circ}$ 95/9626-0) pelo suporte financeiro. LPCM é bolsista de Produtividade em Pesquisa do CNPq. FAGG foi bolsista de doutorado da CAPES. VBZ e RJAS receberam bolsa de doutorado e mestrado do CNPq, respectivamente. 


\section{Referências bibliográficas}

AB’ SABER, A.N. Os domínios morfoclimáticos na América do Sul. Primeira aproximação. Geomorfologia (Inst. Geogr. Univ. S. Paulo) 52: 1-21.

ALMEIDA, F.F.M. 1974. Fundamentos geológicos do relevo paulista. Série Teses e Monografias - 14, USP, São Paulo.

ALMEIDA-SCABBIA, R.J. 1996. Fitossociologia de um trecho de floresta Atlântica no Parque Estadual Intervales, SP. Dissertação de mestrado, Universidade Estadual Paulista, Rio Claro.

APG (Angiosperm Phylogeny Group). 2003. An update of the angiosperm phylogeny group classification for the orders and families of flowering plants: APG II. Bot. J. Linn. Soc. Lond. 141:399-436.

BORGO, M. \& SILVA, S.M. 2003. Epífitos vasculares em fragmentos de Floresta Ombrófila Mista, Curitiba, Paraná, Brasil. Rev. Bras. Bot. 26: 391-401.

DEAN, W. 1995. A ferro e fogo: a história e a devastação da Mata Atlântica brasileira. Companhia das Letras, São Paulo.

DITTRICH, V.A.O., KOZERA, C. \& SILVA, S.M. 1999. Levantamento florístico dos epífitos vasculares do Parque Barigüi, Curitiba, Paraná, Brasil. Iheringia 52: 11-21.

FUNDAÇÃO FLORESTAL. 2001. Intervales: fundação para a conservação e a produção florestal do estado de São Paulo. Secretaria do Meio Ambiente, São Paulo.

GALETTI, M. \& CHIVERS, D.J. 1995. Palm harvest threatens Brazil's best protected area of Atlantic Forest. Oryx 29: 225-226.

GALETTI, M., LAPS, R. \& PIZO, M.A. 2000. Frugivory by toucans (Ramphastidae) at two altitudes in the Atlantic Forest of Brazil. Biotropica 32: 842-850.

GENTRY, A.H. \& DODSON, C.H. 1987. Diversity and biogeography of neotropical vascular epiphytes. Ann. Missouri Bot. Gard. 74: 205-233.

GUILHERME, F.A.G, MORELLATO, L.P.C. \& ASSIS, M.A. 2004. Horizontal and vertical tree community structure in a section of lowland Atlantic Rain Forest in the Intervales State Park, southeastern Brazil. Rev. Bras. Bot. 27(4): 725-737.

IBGE. 1991. Manual técnico da vegetação brasileira. Sériemanuais técnicos em geociências. DEDIT/CDDI, Rio de Janeiro.

IVANAUSKAS, N.M., MONTEIRO, R. \& RODRIGUES, R.R. 2000. Similaridade florística entre áreas de floresta Atlântica no estado de São Paulo. Braz. J. Ecol. 1/2: 71-81.

IVANAUSKAS, N.M., MONTEIRO, R. \& RODRIGUES, R.R. 2001. Levantamento florístico de trecho de floresta Atlântica em Pariquera-Açu, São Paulo, Brasil. Naturalia 26: 97-129.
KAWASAKI, M.L. \& HOLST, B.K. 2002. Two new species of Plinia (Myrtaceae) from coastal forests of Brazil. Brittonia 54: 94-98.

KIM, A.C. 1996. Lianas da Mata Atlântica do Estado de São Paulo. Dissertação de mestrado. Universidade Estadual de Campinas, Campinas.

LEITÃO FILHO, H.F. 1994. Diversity of arboreal species in Atlantic rain forest. An. Acad. Bras. Cienc. 66: 91-96.

LIMA, M.P.M. \& GUEDES-BRUNI, R.R. 1994. (Org.) Reserva Ecológica de Macaé de Cima, Nova Friburgo - RJ: aspectos florísticos das espécies vasculares. Rio de Janeiro, Jardim Botânico, v.1.

MADISON, M. 1977. Vascular epithytes: their systematic occurrence and salient features. Selbyana 2:1-13.

MAMEDE, M.C.H., CORDEIRO, I. ROSSI, L. MELO, M.M.R.F. \& OLIVEIRA, R.J. 2004. Mata Atlântica. In Estação Ecológica Juréia-Itatins: ambiente físico, flora e fauna. (O.A.V. Marques \& W. Duleba, eds.). Holos Editora, Ribeirão Preto, p.115-132.

MANTOVANI, W. 1990. A dinâmica das florestas de encosta Atlântica. In Anais do II Simpósio de Ecossistemas da Costa Sul e Sudeste Brasileira, São Paulo, p.304-313.

MANTOVANI, W. 1998. Dinâmica da Floresta Pluvial Atlântica. In Anais do IV Simpósio de Ecossistemas Brasileiros. ACIESP Águas de Lindóia, p.1-20.

MORELLATO, L.P.C. \& HADDAD, C.F.B. 2000. Introduction: The Brazilian Atlantic Forest. Biotropica 32: 786-792.

MORELLATO, L.P.C. \& LEITÃO-FILHO, H.F. 1996. Reproductive phenology of climbers in a Southeasthern Brazilian forest. Biotropica 28:180-191.

MORELLATO, L.P.C., TALORA, D.C., TAKAHASI, A., BENCKE, C.C., ROMERA, E.C. \& ZIPPARRO, V.B. 2000. Phenology of Atlantic Rain Forest trees: a comparative study. Biotropica 32: 811-823.

MORI, S.A., BOOM, B.M., CARVALHO, A.M. \& SANTOS, T.S. 1983. Ecological importance of Myrtaceae in an Eastern Brazilian wet forest. Biotropica 15:68-70.

MORI, S.A. 1988. Eastern, extra-amazonian Brazil. In Floristic inventory of tropical countries. (D.G. Campbell \& H.D. Hammond, eds.). New York Botanical Garden, New York. p.428-454.

MYERS, N., MITTERMEIER, R.A., MITTERMEIER, C.G., FONSECA, G.A.B. \& KENT, J. 2000. Biodiversity hotspots for conservation priorities. Nature 403: 853-858.

OLIVEIRA FILHO, A.T. \& FONTES, M.A.L. 2000. Patterns of floristic differentiation among Atlantic Forests in Southeastern Brazil, and the influence of climate. Biotropica 32: 793-810. 
PEIXOTO, A.L. \& GENTRY, A.H. 1990. Diversidade e composição florística da mata de tabuleiro na Reserva Florestal de Linhares (Espírito Santo, Brasil). Rev. Bras. Bot. 13:19-25.

PIZO, M.A. 2002. The seed-dispersers and fruit syndromes of Myrtaceae in the brazilian Atlantic Forest. In Seed dispersal and frugivory: ecology, evolution and conservation. (D.J. Levey, W.R. Silva \& M. Galetti, eds.). CAB Publishing, New York. p.129-143.

PIZO, M.A. \& MORELLATO, L.P.C. 2002. A new rain-operated seed dispersal mechanism in Bertolonia mosenii (Melastomataceae), a Neotropical rainforest herb. Am. J. Bot. 89: 169-171.

PIZO, M.A. \& OLIVEIRA, P.S. 2000. The use of fruits and seeds by ants in the Atlantic Forest of southeast Brazil. Biotropica 32: 851-861.

SANCHES, M., PEDRONI, F., LEITÃO FILHO, H.F. \& CÉSAR, O. 1999. Composição florística de um trecho de floresta ripária na Mata Atlântica em Picinguaba, Ubatuba, SP. Rev. Bras. Bot. 22:31-42.

SCARANO, F.R. 2002. Structure, function and floristic relationships of plant communities in stressful habitats marginal to the Brazilian atlantic rainforest. Ann. of Bot. 90: 517-524.

SCARANO, F.R. 2004. O papel da biodiversidade no funcionamento de ecossistemas. In Pesquisa da biodiversidade: princípios, desafios e avanços: a experiência do Programa Biota/Fapesp (T. Lewinsohn, A.C. Joly, M.S. Buckeridge \& L.A. Martinelli, eds.) (no prelo).

SCUDELLER, V.V., MARTINS, F.R. \& SHEPHERD, GJ. 2001. Distribution and abundance of arboreal species in the atlantic ombrophilous dense forest in Southeastern Brazil. Plant Ecol. 152:185-199.

SETZER, J. 1966. Atlas Climático e Ecológico do Estado de São Paulo. Comissão Interestadual da Bacia ParanáUruguai, São Paulo.

SILVA MATOS, D.M. \& BOVI, M.L.A. 2002. Understanding the threats to biological diversity in southeastern Brazil. Biodiversity and Conservation 11:1747-1758.

TABARELLI, M. \& MANTOVANI, W. 1999. A riqueza de espécies arbóreas na floresta atlântica de encosta no estado de São Paulo (Brasil). Rev. Bras. Bot. 22:217-223.

TALORA, D.C. \& MORELLATO, L.P.C. 2000. Fenologia de espécies arbóreas em floresta de planície litorânea do sudeste do Brasil. Rev. Bras. Bot. 23: 13-26.

VIEIRA, E.M. \& IZAR, P. 1999. Interactions between aroids and arboreal mammals in the Brazilian Atlantic rainforest. Plant Ecol. 145: 75-82.

ZIPPARRO, V.B. 2004. Fenologia reprodutiva de espécies arbóreas em área de Floresta Atlântica no Parque Estadual Intervales - Base Saibadela, Sete Barras, SP. Tese de doutorado, Universidade Estadual Paulista, Rio Claro.
Título: Levantamento Florístico de Floresta Atlântica no Sul do Estado de São Paulo, Parque Estadual Intervales, Base Saibadela

Autores: Valesca Bononi Zipparro, Frederico Augusto G. Guilherme, Renata J. Almeida-Scabbia \& L. Patrícia C. Morellato

Biota Neotropica, Vol. 5( número 1): 2005

http://www.biotaneotropica.org.br/v5n1/pt/ abstract?inventory+BN02605012005

Recebido: 01/10/2004

Revisado: 13/12/2004

Publicado: 01/01/2005

ISSN 1676-0603 
Tabela 1. Listagem das fanerógamas encontradas na Floresta Atlântica da Base Saibadela, no Parque Estadual Intervales, Sete Barras-SP. As espécies são divididas em eudicotiledôneas (incluindo os grupos parafiléticos) e monocotiledôneas, em ordem alfabética de famílias. São fornecidos os nomes comuns, a forma de vida (ou hábito) - árvore (arv), liana (lian), arbusto (arb), herbácea (herb), epífita (epf), estranguladora (est) e parasita (par) -, o modo de dispersão e o número de registro no HRCB, para o material fértil. Algumas espécies sem número de registro representam coletas de material estéril ou apenas observadas no campo.

\section{\begin{tabular}{l} 
Família / Espécie \\
\hline EUDICOTILEDÔNEAS*
\end{tabular}}

Acanthaceae

Aphelandra liboniana Linden ex Hook. $\mathrm{f}$.

Aphelandra ornata (Nees) T. Anderson

Justicia carnea Lindl.

Acanthaceae

Amaranthaceae

Celosia brasiliensis Moq.

Hebanthe paniculata Mart.

Anacardiaceae

Tapirira guianensis Aubl.

Annonaceae

Duguetia sp.

Guatteria australis A. St. Hill.

Rollinia cf. dolabripetala (Raddi) R.E. Fr.

Rollinia sericea R.E.Fr.

Xylopia cf. brasiliensis Spreng.

Apocynaceae

Aspidosperma cf. compactinervium Kuhlm.

Malouetia cestroides Muell. Arg.

Tabernaemontana sp.

Aquifoliaceae

Ilex amara (Vell.) Loes

Ilex theazans Mart.

Araliaceae

Dendropanax sp.

Oreopanax capitatus (Jacq.) Decne. \& Planch.

Schefflera angustissima (Marchal) Frodin

Aristolochiaceae

Aristolochia paulistana Hoehne
Nome comum

Hábito Dispersão HRCB

$\begin{array}{llll}\text { - } & \text { arb } & \text { auto } & 21286 \\ \text { - } & \text { herb } & \text { auto } & 25527 \\ \text { Bálsamo } & \text { arb } & \text { auto } & 25112 \\ \text { - } & \text { herb } & - & 35582\end{array}$

Espora-de-galo

herb auto

lian anemo

arv

zoo

$\begin{array}{llll}\text { - } & \text { arv } & \text { zoo } & 25188 \\ \text { Pindaúva-branca } & \text { arv } & \text { zoo } & 21294 \\ \text { - } & \text { arv } & \text { zoo } & - \\ \text { Araticum } & \text { arv } & \text { zoo } & 21287 \\ \text { Pindaúva-vermelha } & \text { arv } & \text { zoo } & 27807\end{array}$

Peroba

arv

anemo

21650

Guairana

arv

auto

35564

$-$

arb

zoo

27766

$-$

arv

zoo

25502

- $\quad \operatorname{arv}$

zoo

$\begin{array}{llll}- & \text { arb } & \text { zoo } & 25030 \\ \text { - } & \text { est } & \text { zoo } & 21785 \\ \text { Rameira } & \text { arv } & \text { zoo } & 21329\end{array}$

Mil-homens

lian auto

21297 
Tabela 1. Continuação

\begin{tabular}{|c|c|c|c|c|}
\hline Família / Espécie & Nome comum & Hábito & Dispersão & $\begin{array}{l}\mathrm{HRCB} \\
\end{array}$ \\
\hline \multicolumn{5}{|l|}{ Asteraceae } \\
\hline Mikania conferta Gardn. & - & lian & anemo & 25024 \\
\hline Mikania sp. & - & lian & anemo & 21336 \\
\hline Piptocarpha macropoda (DC.) Baker & - & arv & anemo & 21337 \\
\hline Vernonia petiolaris DC. & Fumão & arv & anemo & 21303 \\
\hline Vernonia puberula Less. & Fumão & arv & anemo & 21302 \\
\hline \multicolumn{5}{|l|}{ Balanophoraceae } \\
\hline Lophophyton sp. & Flor-de-ingaeiro & par & - & 24804 \\
\hline \multicolumn{5}{|l|}{ Begoniaceae } \\
\hline Begonia capanemae Brade & Begonia & herb & anemo & 21366 \\
\hline Begonia convolvulaceae A.DC. & Begonia & lian & anemo & 21298 \\
\hline Begonia lanceolata Vell. & Begonia & epf & anemo & 21641 \\
\hline Begonia radicans Vell. & Begonia & lian & anemo & 21330 \\
\hline Begonia sp. 1 & Begonia & epf & anemo & 35959 \\
\hline Begonia sp. 2 & - & epf & anemo & 35566 \\
\hline \multicolumn{5}{|l|}{ Bignoniaceae } \\
\hline Adenocalymma hatschbachii A. H. Gentry & - & lian & anemo & 25283 \\
\hline Clytostoma cf. sciuripabulum Bureau \& K. Schum. & - & lian & anemo & 35708 \\
\hline Jacaranda cf. montana Morawetz & Carova & $\operatorname{arv}$ & anemo & 21661 \\
\hline Parabignonia unguiculata (Vell.) R. H. Gentry & - & lian & anemo & 25179 \\
\hline Schlegelia parviflora (Oerst.) Monach & - & lian & zoo & 21878 \\
\hline Tabebuia serratifolia (Vahl) G. Nicholson & Ipê-amarelo & arv & anemo & 21635 \\
\hline \multicolumn{5}{|l|}{ Boraginaceae } \\
\hline Cordia ecalyculata Vell. & - & arv & zoo & - \\
\hline Cordia sylvestris Fresen. & Juretê & arv & zoo & 21367 \\
\hline \multicolumn{5}{|l|}{ Burseraceae } \\
\hline Protium sp. & Mesca & arv & zoo & 36438 \\
\hline \multicolumn{5}{|l|}{ Cactaceae } \\
\hline Rhipsalis elliptica G. Lindb. ex K. Schum. & - & epf & zoo & 21884 \\
\hline Rhipsalis pachyptera Pfeiff. & - & epf & zoo & 36274 \\
\hline Rhipsalis teres (Vell.) Steud. & - & epf & zoo & 21883 \\
\hline \multicolumn{5}{|l|}{ Canellaceae } \\
\hline Cinnamodendron dinisii Schwacke & Pimenta & arv & zoo & 25503 \\
\hline \multicolumn{5}{|l|}{ Cannabaceae } \\
\hline Trema micrantha (L.) Blume & Crindiúva & arv & zoo & 21659 \\
\hline \multicolumn{5}{|l|}{ Caricaceae } \\
\hline Jacaratia spinosa (Aubl.) A. DC. & Jaracatiá & arv & zoo & - \\
\hline
\end{tabular}


Tabela 1. Continuação

Família / Espécie

Nome comum

Hábito Dispersão HRCB

Celastraceae

Maytenus aquifolium Mart.

Maytenus cf. alaternoides Reissek

Maytenus communis Reissek

Maytenus ligustrina Reissek

Maytenus robusta Reissek

Maytenus schumanniana Loes.

Maytenus sp. 1

Maytenus sp. 2

Maytenus sp. 3

Chloranthaceae

Hedyosmum brasiliense Miq.

Chrysobalanaceae

Hirtella hebeclada Moric. ex DC.

Parinari excelsa Sabine

Clusiaceae

Clusia parviflora Humb. \& Bonpl. ex Willd.

Garcinia gardneriana (Planch. \& Triana) Zappi

Combretaceae

Combretum sp.

Terminalia sp.

Cucurbitaceae

Cucurbitaceae 1

Cucurbitaceae 2

Elaeocarpaceae

Sloanea guianensis (Aubl.) Benth.

Sloanea monosperma Vell.

Erythroxylaceae

Erythroxylum ambiguum Peyr.

Euphorbiaceae

Alchornea glandulosa Poepp.

Alchornea triplinervia (Spreng.) Müll. Arg.

Aparisthmium cordatum (A. Juss.) Baill.

Gymnanthes concolor (Spreng.) Muell. Arg.

Maprounea guianensis Aubl.

Pera glabrata (Schott) Poepp. ex Baill.

Tetrorchidium rubrivenium Poepp.

$\begin{array}{llll}\text { Espinheira-santa } & \text { arv } & \text { zoo } & 21881 \\ \text { - } & \text { arv } & \text { zoo } & 36349 \\ \text { - } & \text { arv } & \text { zoo } & - \\ \text { - } & \text { arv } & \text { zoo } & 21875 \\ \text { Pau-d'arco } & \text { arv } & \text { zoo } & 21877 \\ \text { - } & \text { arv } & \text { zoo } & 21873 \\ \text { - } & \text { arv } & \text { zoo } & 21870 \\ \text { - } & \text { arv } & \text { zoo } & 21625 \\ \text { - } & \text { arv } & \text { zoo } & 21890\end{array}$

arv $\quad$ zoo

arv

zOO

arv

ZOO

$-$

Bacupari

epf

zOO

arv

ZOO

- Guarajuva

lian

anemo

arv

anemo

25193

Pepino-de-rato

lian

zoo

25432

lian zoo

Nimbiúva

arv

zoO

21306

Pau-ferro

arv

ZOO

21305

$-\quad \operatorname{arv}$

zoO

35817

Tapiá

$\operatorname{arv}$

zoo

21888

Tapiá

arv

zOO

21345

Quineira

arv

ZOO

35568

$-$

arv

auto

21343

$-$

Tabocuva

arv

Z0O

arv

zoO

25280

Caxeta

arv

Z0O

21349 
Tabela 1. Continuação

\begin{tabular}{|c|c|c|c|c|}
\hline Família / Espécie & Nome comum & Hábito & Dispersão & HRCB \\
\hline \multicolumn{5}{|l|}{ Fabaceae - Caesalpinioideae } \\
\hline Bauhinia sp. & Escada-de-macaco & lian & auto & - \\
\hline Copaifera langsdorfii Desf. & Copaíba-vermelha & arv & zoo & 21862 \\
\hline Copaifera trapezifolia Hayne & Copaíba-branca & arv & zoo & 25022 \\
\hline Hymenaea courbaril var. altissima (Ducke) Y.T. Lee \& Langenh. & Jatobá & arv & zoo & 21744 \\
\hline Schizolobium parahyba (Vell.) S.F. Blake & Guapiruvu & arv & auto & - \\
\hline Sclerolobium denudatum Vogel & - & arv & anemo & 38115 \\
\hline \multicolumn{5}{|l|}{ Fabaceae - Faboideae } \\
\hline Andira cf. fraxinifolia Benth. & - & arv & zoo & - \\
\hline Andira cf. ormosioides Benth. & - & arv & zoo & 21841 \\
\hline Dahlstedtia pinnata (Taub.) Burkart & Caracatinga & arv & auto & 21638 \\
\hline Dalbergia frutescens (Vell.) Britton & - & arv & anemo & 25190 \\
\hline Lonchocarpus cultratus (Vell.) A.M.G. Azevedo \& H.C. Lima & - & arv & anemo & 21832 \\
\hline Machaerium lanceolatum (Vell.) J.F. Macbr. & marmeleiro & lian/arv & anemo & 25025 \\
\hline Mucuna cf. urens (L.) Medik. & Olho-de-boto & lian & auto & - \\
\hline Myrocarpus frondosus Allemao & Cabreúva & arv & anemo & 21831 \\
\hline Ormosia cf. arborea (Vell.) Harms & Olho-de-cabra & arv & zoo & - \\
\hline Platymiscium floribundum Vogel & Jacaranda & arv & anemo & - \\
\hline Pterocarpus rohrii Vahl & Pau-sangue & arv & anemo & 21397 \\
\hline Swartzia flaemingii Raddi & Pacová-de-macaco & arv & zoo & 21851 \\
\hline Vigna sp. & Feijão-bravo & lian & - & 25032 \\
\hline Zollernia ilicifolia (Brongn.) Vogel & - & arv & zoo & 35563 \\
\hline \multicolumn{5}{|l|}{ Fabaceae - Mimosoideae } \\
\hline Inga edulis Mart. & Ingá-feijão & arv & zoo & 21391 \\
\hline Inga marginata Willd. & Ingá-mirim & arv & zoo & 21825 \\
\hline Inga sessilis (Vell.) Mart. & Ingá-ferradura & arv & zoo & 21313 \\
\hline Inga sp. & - & arv & zoo & 27755 \\
\hline Pseudopiptadenia warmingii (Benth.) G.P.Lewis \& M.P.Lima & Caovi & arv & anemo & 21390 \\
\hline \multicolumn{5}{|l|}{ Gesneriaceae } \\
\hline Codonanthe devosiana Lem. & - & epf & zoo & 36282 \\
\hline Codonanthe gracilis (Mart.) Hanst. & - & lian & zoo & 25185 \\
\hline Nematanthus jolyanus (Handro) A. Chautems & - & epf & zoo & 35952 \\
\hline Sinningia sp. & - & herb & - & 25111 \\
\hline \multicolumn{5}{|l|}{ Hippocrateaceae } \\
\hline Cheiloclinium cf. cognatum (Miers) A.C. Sm. & - & lian & zoo & 21782 \\
\hline Peritassa sp. & - & arv & zoo & 36283 \\
\hline Salacia elliptica (Mart. ex Schult.) G. Don & - & lian & zoo & 25191 \\
\hline
\end{tabular}


Tabela 1. Continuação

\begin{tabular}{|c|c|c|c|c|}
\hline Família / Espécie & Nome comum & Hábito & Dispersão & HRCB \\
\hline \multicolumn{5}{|l|}{ Icacinaceae } \\
\hline Citronella megaphylla (Miers.) Howard & - & arv & $\mathrm{zOO}$ & 21355 \\
\hline \multicolumn{5}{|l|}{ Lamiaceae } \\
\hline Lamiaceae & - & herb & - & 35581 \\
\hline \multicolumn{5}{|l|}{ Lauraceae } \\
\hline Cryptocaria moschata Nees \& Martius ex Nees & Canela- nhutinga & arv & zoo & 21312 \\
\hline Cryptocarya sp. & - & arv & zoo & 21788 \\
\hline Endlicheria paniculata (Spreng.) J.F. Macbr. & Canela-fogo & arv & zoo & 21311 \\
\hline Licaria armeniaca (Nees) Kosterm. & - & arv & zoo & 38114 \\
\hline Nectandra megapotamica (Spreng.) Mez & Canela & arv & zoo & 35833 \\
\hline Nectandra membranacea (Sw.) Griseb. & - & arv & zoo & 21768 \\
\hline Nectandra saligna Nees & - & arv & zoo & 21469 \\
\hline Nectandra sp. & - & arv & zoo & 21830 \\
\hline Ocotea aciphylla (Nees) Mez & - & arv & zoo & 21359 \\
\hline Ocotea dispersa (Nees) Mez & Canela-fogo & arv & zoo & 21767 \\
\hline Ocotea elegans Mez & Canela-sassafrás & arv & zoo & 21356 \\
\hline Ocotea tabacifolia (Meisn.) Rohwer & Canela-inhuva & arv & zoo & 25103 \\
\hline Ocotea teleiandra (Meisn.) Mez & Canela-fogo & arv & zoo & 21291 \\
\hline Lauraceae & - & arv & zoo & 21649 \\
\hline
\end{tabular}

Lecythidaceae

Cariniana estrellensis (Raddi) Kuntze

Jequitibá

arv anemo

Loganiaceae

Spigelia sp.

Strychnos brasiliensis (Spreng.) Mart.

$\begin{array}{llll}\text { - } & \text { herb } & - & 32374 \\ \text { - } & \text { arb } & \text { zoo } & 21863\end{array}$

Strychnos trinervis (Vell.) Mart.

Espora-de-galo

lian

zoo

27802

Magnoliaceae

Talauma ovata A. St.-Hil.

Pinha-brava

arv

ZOO

21400

Malpighiaceae

Bunchosia fluminensis Griseb.

Heteropterys intermedia (Grisebach) A. Juss.

Heteropterys sp.

$\begin{array}{llll}\text { - } & \text { arv } & \text { zoo } & 35788 \\ \text { - } & \text { lian } & \text { anemo } & 35859 \\ \text { - } & \text { lian } & \text { anemo } & 36319 \\ \text { - } & \text { lian } & \text { anemo } & 21392 \\ \text { Batata-brava } & \text { lian } & \text { anemo } & 25528\end{array}$

Hiraea sp.

Stigmaphyllon sp.

Batata-brava

Embiruçu

arv anemo

Pseudobombax sp.

Mata-pau

est anemo

Malvaceae

arb

35958 
Tabela 1. Continuação

\begin{tabular}{|c|c|c|c|c|}
\hline Família / Espécie & Nome comum & Hábito & Dispersão & HRCB \\
\hline \multicolumn{5}{|l|}{ Marcgraviaceae } \\
\hline Marcgravia polyantha Delpino & Hera-de-árvore & lian & zoo & 21314 \\
\hline Norantea cf. brasiliensis Choisy & - & lian & zoo & - \\
\hline \multicolumn{5}{|l|}{ Melastomataceae } \\
\hline Bertolonia mosenii Cogn. & - & herb & hidro & 21552 \\
\hline Clidemia blepharodes D.C. & - & lian & zoo & 21834 \\
\hline Clidemia hirta (L.) D. Don & - & herb & zoo & 21404 \\
\hline Henriettella glabra Cogn. & - & arv & zoo & - \\
\hline Leandra dasytricha (A. Gray) Cog. & - & arv & zoo & 26346 \\
\hline Leandra glazioviana Cogn. & - & arb & zoo & 21857 \\
\hline Leandra mosenii Cogn. & - & arb & zoo & 21401 \\
\hline Leandra reversa (DC.) Cogn. & - & arb & zoo & 21802 \\
\hline Leandra sp. & - & arv & zoo & 21316 \\
\hline Miconia cabussu Hoehne & Pixiricuçú & arv & zoo & - \\
\hline Miconia cinnamomifolia (DC.) Naudin & Suvi-suvi & arv & zoo & 35753 \\
\hline Miconia dodecandra Cogn. & - & arv & zoo & 21612 \\
\hline Miconia holosericea (L.) DC. & - & arv & zoo & 35999 \\
\hline Miconia latecrenata Triana & - & arb & zoo & 21786 \\
\hline Miconia rigidiuscula Cogn. & - & arv & zoo & - \\
\hline Miconia sp. 1 & - & arv & zoo & 21405 \\
\hline Miconia sp. 2 & - & arv & zoo & 25199 \\
\hline Miconia tristis ssp. australis Weirdock & - & arv & zoo & 21319 \\
\hline Mouriri chamissoana Cogn. & - & arv & zoo & 35754 \\
\hline Ossaea retropila Triana & - & arb & zoo & 21855 \\
\hline Ossaea sanguinea Cogn. & - & arb & zoo & 21324 \\
\hline Salpinga cf. longifolia (Cham.) Triana & - & herb & hidro & 35846 \\
\hline Tibouchina regnellii Cogn. & Jacatirão & arv & anemo & - \\
\hline \multicolumn{5}{|l|}{ Meliaceae } \\
\hline Cabralea canjerana (Vell.) Mart. & Canjerana & arv & zoo & 16579 \\
\hline Cedrela fissilis Vell. & Cedro & arv & anemo & - \\
\hline Guarea macrophylla Vahl & Café-bravo & arv & zoo & 21406 \\
\hline Trichilia lepidota Mart. & - & arv & zoo & 21842 \\
\hline Trichilia cf. pallens C. DC. & - & arv & zoo & 25184 \\
\hline \multicolumn{5}{|l|}{ Mendonciaceae } \\
\hline Mendoncia velloziana Mart. & - & lian & zoo & 35831 \\
\hline \multicolumn{5}{|l|}{ Menispermaceae } \\
\hline Abuta selloana Eichler & & lian & zoo & 21828 \\
\hline Hyperbaena sp. & - & lian & zoo & 21409 \\
\hline
\end{tabular}


Tabela 1. Continuação

\begin{tabular}{|c|c|c|c|c|}
\hline Família / Espécie & Nome comum & Hábito & Dispersão & HRCB \\
\hline \multicolumn{5}{|l|}{ Monimiaceae } \\
\hline Mollinedia cf. oligantha Perkins & - & arv & zoo & 25088 \\
\hline Mollinedia schottiana (Spreng.) Perkins & - & arv & zoo & 21459 \\
\hline Mollinedia aff. triflora (Spreng.) Tul. & - & arv & zoo & 21843 \\
\hline Mollinedia uleana Perkins & - & arv & zoo & 21410 \\
\hline \multicolumn{5}{|l|}{ Moraceae** } \\
\hline Brosimum cf. glazioui Taub. & - & arv & zoo & 36315 \\
\hline Brosimum cf. guianense (Aubl.) Huber & - & arv & zoo & 35822 \\
\hline Dorstenia sp. & - & herb & zoo & 25183 \\
\hline Ficus enormis (Mart. ex Miquel) Miquel & - & arv & zoo & 21754 \\
\hline Ficus gomelleira Kunth. & - & arv & zoo & 21761 \\
\hline Ficus insipida Willd. & Figueira-branca & arv & zoo & 16580 \\
\hline Ficus obtusiuscula Miq. & Figueira & arv & zoo & - \\
\hline Ficus trigonata Linnaeus & - & arv & zoo & 21762 \\
\hline \multicolumn{4}{|l|}{ (Baill.) Burger } & 21495 \\
\hline \multicolumn{5}{|l|}{ Myristicaceae } \\
\hline Virola bicuhyba (Schott. ex A. DC.) Warb. & Bocuva-mirim & arv & zoo & 21823 \\
\hline Virola gardneri (A. DC.) Warb. & Bocuva-açu & arv & zoo & 21494 \\
\hline \multicolumn{5}{|l|}{ Myrsinaceae } \\
\hline Cybianthus cf. cuneifolius Mart. & - & arb & zoo & 25097 \\
\hline Rapanea umbellata Mart. & Carvalho & arv & zoo & 21610 \\
\hline Stylogyne ambigua Mez & - & arv & zoo & 27765 \\
\hline \multicolumn{5}{|l|}{ Myrtaceae } \\
\hline Calycorectes acutatus (Miq.) Toledo & - & arv & zoo & 21502 \\
\hline Calycorectes australis D. Legrand & Uvaia & arv & zoo & 25048 \\
\hline Calyptranthes lanceolata O. Berg. & - & arv & zoo & 21501 \\
\hline Campomanesia guaviroba (DC.) Kiaersk. & - & arv & zoo & 25434 \\
\hline Campomanesia neriiflora (O. Berg.) Nied. & Guabiroba & arv & zoo & 27808 \\
\hline Campomanesia schlechtendhaliana (O. Berg.) Nied. & Guabiroba & arv & zoo & 35577 \\
\hline Campomanesia xanthocarpa O. Berg. & Guabiroba & arv & zoo & 27808 \\
\hline Eugenia beaurepairiana (Kiaersk.) D. Legrand & - & arv & zoo & - \\
\hline Eugenia bocainensis Mattos & Jambro & arv & zoo & 21414 \\
\hline Eugenia cambucarana Kiaersk. & Uvaia & arv & zoo & 21654 \\
\hline Eugenia capitulifera O. Berg. & - & arv & zoo & 25074 \\
\hline Eugenia cerasiflora Miq. & - & arv & zoo & 36286 \\
\hline Eugenia cf. cereja D. Legrand & - & arv & zoo & 35737 \\
\hline Eugenia cf. convexinervia D. Legrand & - & arv & zoo & 35734 \\
\hline
\end{tabular}


Tabela 1. Continuação

\begin{tabular}{|c|c|c|c|c|}
\hline Família / Espécie & Nome comum & Hábito & Dispersão & HRCB \\
\hline \multicolumn{5}{|l|}{ Myrtaceae (cont.) } \\
\hline Eugenia cuprea (O. Berg.) Nied. & Murtinha & arv & zoo & 21413 \\
\hline Eugenia handroana D. Legrand & Camarinha & arv & zoo & 21774 \\
\hline Eugenia melanogyna (D. Legrand) Sobral & - & arv & zoo & 21412 \\
\hline Eugenia mosenii (Kausel) Sobral & Cuxita, Jambro & arv & zoo & 21442 \\
\hline Eugenia multicostata D. Legrand & Araça-piranga & arv & zoo & 21664 \\
\hline Eugenia neoglomerata Sobral & Araçá-branco & arv & zoo & 35580 \\
\hline Eugenia neoverrucosa D. Legrand & - & arv & zoo & 21422 \\
\hline Eugenia oblongata O.Berg. & Jambro & arv & zoo & 21423 \\
\hline Eugenia cf. platysema O. Berg. & - & arv & zoo & 25064 \\
\hline Eugenia pruinosa D. Legrand & - & arv & zoo & 36321 \\
\hline Eugenia riedeliana O. Berg. & - & arv & zoo & 25063 \\
\hline Eugenia speciosa Camb. & - & arv & zoo & 21441 \\
\hline Eugenia stictosepala Kiaersk. & - & arv & zoo & 21617 \\
\hline Eugenia subavenia O. Berg. & - & arv & zoo & 21424 \\
\hline Eugenia sp. 1 & Araçá-branco & arv & zoo & 25076 \\
\hline Eugenia sp. 2 & - & arv & zoo & 21787 \\
\hline Eugenia sp. 3 & - & arv & zoo & 32209 \\
\hline Eugenia sp. 4 & - & arv & zoo & 25078 \\
\hline Gomidesia anacardiaefolia (Gardner) O. Berg. & - & arv & zoo & 21419 \\
\hline Gomidesia flagellaris D. Legrand & - & arv & zoo & 21784 \\
\hline Gomidesia spectabilis (DC.) O. Berg. & - & arv & zoo & 21421 \\
\hline Gomidesia tijucensis Kiaersk. & - & arv & zoo & 25072 \\
\hline Marlierea eugeniopsoides (D. Legrand \& Kausel) D. Legrand. & - & arv & zoo & 21506 \\
\hline Marlierea obscura O. Berg. & Goiaba & arv & zoo & 21432 \\
\hline Marlierea regeliana O. Berg. & - & arv & zoo & 21865 \\
\hline Marlierea aff. strigipes O. Berg. & - & arv & zoo & 36017 \\
\hline Marlierea suaveolens Cambess. & - & arv & zoo & 21836 \\
\hline Marlierea tomentosa Cambess. & Vapurunga & arv & zoo & 21415 \\
\hline Myrceugenia campestris (D.C.) D. Legrand \& Kausel & - & arv & zoo & 21444 \\
\hline Myrceugenia kleinii D. Legrand \& Kausel & - & arv & zoo & 25056 \\
\hline Myrceugenia myrcioides (Cambess.) O. Berg. & Pau-d’arco & arv & zoo & 21483 \\
\hline Myrceugenia reitzii D. Legrand \& Kausel & - & arv & zoo & 21772 \\
\hline Myrcia macrocarpa D.C. & - & $\operatorname{arv}$ & zoo & 21806 \\
\hline Myrcia pubipetala Miq. & - & arv & zoo & 32220 \\
\hline Myrcia tenuivenosa Kiaersk. & - & arv & zoo & 35741 \\
\hline Myrcia sp. & - & arv & zoo & 21582 \\
\hline Myrciaria floribunda (West ex Willd.) O. Berg. & Uvaia & arv & zoo & 25438 \\
\hline
\end{tabular}


Tabela 1. Continuação

\begin{tabular}{|c|c|c|c|c|}
\hline Família / Espécie & Nome comum & Hábito & Dispersão & HRCB \\
\hline \multicolumn{5}{|l|}{ Myrtaceae (cont.) } \\
\hline Myrciaria tenella (DC.) O. Berg. & - & $\operatorname{arv}$ & zoo & 36320 \\
\hline Neomitranthes glomerata (D. Legrand) D. Legrand & - & arv & zoo & 21758 \\
\hline Plinia complanata M.L.Kawasaki \& B.Holst & - & arv & zoo & 35555 \\
\hline Plinia pauciflora M.L.Kawasaki \& B.Holst & - & arv & zoo & 31494 \\
\hline \multicolumn{5}{|l|}{ Nyctagynaceae } \\
\hline Guapira opposita (Vell.) Reitz & Carne-de-vaca & arv & zoo & 21607 \\
\hline Pisonia ambigua Heimerl & - & arv & zoo & 35970 \\
\hline \multicolumn{5}{|l|}{ Olacaceae } \\
\hline Heisteria silvianii Schwacke & - & $\operatorname{arv}$ & zoo & 22232 \\
\hline Tetrastylidium grandifolium (Baill.) Sleumer & Mandigaú & $\operatorname{arv}$ & zoo & 22233 \\
\hline \multicolumn{5}{|l|}{ Onagraceae } \\
\hline Fuchsia regia (Vell.) Munz & - & lian & zoo & 35923 \\
\hline \multicolumn{5}{|l|}{ Passifloraceae } \\
\hline Passiflora sp. 1 & - & lian & $\mathrm{zOO}$ & 35700 \\
\hline Passiflora sp. 2 & Maracujá & lian & zoo & 25284 \\
\hline Passiflora sp. 3 & Maracujá & lian & zoo & 25437 \\
\hline Passiflora sp. 4 & - & lian & zoo & 25435 \\
\hline \multicolumn{5}{|l|}{ Phyllanthaceae } \\
\hline Hyeronima alchorneoides Allemao & Urucurana & $\operatorname{arv}$ & zoo & 21346 \\
\hline Margaritaria nobilis L. f. & - & arv & zoo & 21880 \\
\hline \multicolumn{5}{|l|}{ Phytolaccaceae } \\
\hline Phytolacca dioica L. & Umbueiro & $\operatorname{arv}$ & zoo & 21528 \\
\hline \multicolumn{5}{|l|}{ Picramniaceae } \\
\hline Picramnia gardneri ssp. gardneri Planch. & - & $\operatorname{arv}$ & zoo & 21852 \\
\hline \multicolumn{5}{|l|}{ Piperaceae } \\
\hline Ottonia cf. leptostachya Kunth & - & arb & zoo & 21529 \\
\hline Peperomia glabella Sw. Var. glabella & - & epf & zoo & 21530 \\
\hline Peperomia pseudo-estrellensis C. DC. & - & herb & zoo & 21805 \\
\hline Peperomia sp. 1 & - & epf & zoo & 27805 \\
\hline Peperomia sp. 2 & - & epf & zoo & 27804 \\
\hline Peperomia sp. 3 & - & epf & zoo & 36300 \\
\hline Piper aduncum L. & - & arb & zoo & 21531 \\
\hline Piper cernuum Vell. & - & arb & $\mathrm{zoO}$ & 21484 \\
\hline Piper sp. 1 & - & arb & zoo & 21807 \\
\hline Piper sp. 2 & - & epf & zoo & 35744 \\
\hline Piperaceae & - & epf & zoo & 36302 \\
\hline
\end{tabular}


Tabela 1. Continuação

Família / Espécie

Polygonaceae

Ruprechtia laxiflora Meisn.

Proteaceae

Roupala brasiliensis Klotzsch

Quiinaceae

Quiina glaziovii Engl. in Mart.

Rhamnaceae

Rhamnidium elaeocarpum Reissek

Rhamnaceae

Rubiaceae

Alibertia myrciifolia Schum.

Alseis floribunda Schott

Amaioua guianensis Aubl.

Bathysa australis (St. Hil.) Benth. \& Hook. f.

Chomelia sp.

Coccocypselum krauseanum Standl.

Coccocypselum sp. 1

Coccocypselum sp. 2

Coccocypselum sp. 3

Coussarea contracta (Walp.) Müll. Arg.

Faramea multiflora A. Rich. ex DC.

Faramea sp. 1

Faramea sp. 2

Geophila repens (L.) I. M. Johnst.

Hillia cf. illustris (Vell.) K. Schumann

Ixora burchelliana Müll. Arg.

Manettia luteo-rubra (Vell.) Benth.

Posoqueria latifolia (Rudge) Roem. \& Schult.

Psychotria astrellantha Wernham

Psychotria birotula L. B. Sm. \& Downs

Psychotria carthagenensis Jacq.

Psychotria leiocarpa Cham. \& Schltr.

Psychotria mapourioides DC.

Psychotria sessilis (Vell.) Müll. Arg.

Psychotria suterella Muell. Arg.

Psychotria umbellata Vell.

Psychotria sp.

Randia nitida (Kunth) DC.
Nome comum

Hábito Dispersão HRCB

arv

anemo

21527

Carne-de-vaca

arv

anemo

Bajaruvá

arv

zoo

21558

arv

ZOO

21449

lian

21808

Tarumã-branco

arv

ZOO

21490

arv

25108

$-$

Cavavana

arv

zoo

21489

21452$$
-
$$$$
-
$$$$
\text { - }
$$$$
-
$$$$
-
$$

Guatambu

arv

auto

35704

arv zoo

25086

herb $\quad$ zoo

herb zoo

35838

35839

21488

25029

25174

21653

32375

36307

21601

Laranja-de-macaco

lian

21567

arv

zoO

21567

21458

25087

-

$-$

Caixeta

arb

zoo

21651

arv

25028

21674

$$
-
$$

Erva-de-anta

arv

zoO

25075

arv

zoo

21486

arv zoo

21603

arb

zoo

35810

arb

ZOO

27803 
Tabela 1. Continuação

\begin{tabular}{|c|c|c|c|c|}
\hline Família / Espécie & Nome comum & Hábito & Dispersão & HRCB \\
\hline \multicolumn{5}{|l|}{ Rubiaceae (cont.) } \\
\hline Rudgea jasminoides ssp. jasminoides (Cham.) Muell. Arg. & Guatambu & $\operatorname{arv}$ & $\mathrm{zOO}$ & 21549 \\
\hline Rudgea recurva Müll. Arg. & Guatambuzinho & arv & zoo & 21446 \\
\hline Rudgea sp. & - & $\operatorname{arv}$ & $\mathrm{zOO}$ & 21668 \\
\hline Simira cf. sampaioana (Standley) Steyerm. & - & arv & anemo & 21790 \\
\hline \multicolumn{5}{|l|}{ Rutaceae } \\
\hline Pilocarpus pauciflorus A. St.-Hil. & - & arv & auto & 21809 \\
\hline Zanthoxylum rhoifolium Lam. & Betari & arv & zoo & - \\
\hline \multicolumn{5}{|l|}{ Sabiaceae } \\
\hline Meliosma sinuata Urb. & - & $\operatorname{arv}$ & zoo & 21671 \\
\hline \multicolumn{5}{|l|}{ Salicaceae } \\
\hline Casearia decandra Jacq. & Conguinho & arv & zoo & 25107 \\
\hline Casearia sp. & - & arv & $\mathrm{zoO}$ & 21625 \\
\hline Xylosma glaberrima Sleumer & Espinho-de-judeu & arv & zoo & 32192 \\
\hline Salicaceae & - & arb & zoo & 21574 \\
\hline \multicolumn{5}{|l|}{ Sapindaceae } \\
\hline Allophylus petiolatus Radlk. & Aperta-guela & $\operatorname{arv}$ & zoo & 21542 \\
\hline Cupania oblongifolia Mart. & Cuvatã & arv & zoo & 21812 \\
\hline Matayba guianensis Aubl. & - & arv & zoo & 21791 \\
\hline Matayba juglandifolia Radlk. & - & $\operatorname{arv}$ & zoo & - \\
\hline Paullinia carpopoda Cambess. & - & lian & zoo & 35601 \\
\hline Paullinia sp. & - & lian & zoo & - \\
\hline Paullinia trigonia Vell. & - & lian & zoo & 32295 \\
\hline \multicolumn{5}{|l|}{ Sapotaceae } \\
\hline Chrysophyllum gonocarpum (Mart. \& Eichl.) Engl. & - & $\operatorname{arv}$ & zoo & 25038 \\
\hline Chrysophyllum inornatum Mart. & Aleixo & arv & zoo & 21588 \\
\hline Chrysophyllum viride Mart. \& Eichler & Bujueiro & arv & zoo & 21813 \\
\hline Diploon cuspidatum (Hoehne) Cronquist & - & arv & zoo & 25096 \\
\hline Ecclinusa ramiflora Mart. & Guacá & $\operatorname{arv}$ & zoo & 27762 \\
\hline Pouteria caimito (Ruiz \& Pav.) Radlk. & Guapeva & arv & zoo & 21798 \\
\hline Pouteria grandifolia (Wall.) Baehni & Guapeva & arv & zoo & 27838 \\
\hline Pouteria psammophila (Mart. ) Radlk. & - & $\operatorname{arv}$ & zoo & 25672 \\
\hline Pouteria venosa (Mart.) Baehni & Guapeva & $\operatorname{arv}$ & zoo & 21481 \\
\hline \multicolumn{5}{|l|}{ Solanaceae } \\
\hline Brunfelsia pauciflora (Cham. \& Schltdl.) Benth. & - & arb & zoo & 21623 \\
\hline Cestrum amictum Schl. & - & arb & zoo & 21620 \\
\hline Cestrum sp. & - & arb & zoo & 21756 \\
\hline Solanum diploconos (Mart.) Bohs & - & arv & zoo & 21662 \\
\hline
\end{tabular}


Tabela 1. Continuação

\begin{tabular}{|c|c|c|c|c|}
\hline Família / Espécie & Nome comum & Hábito & Dispersão & HRCB \\
\hline \multicolumn{5}{|l|}{ Solanaceae (cont.) } \\
\hline Solanum glomuliflorum Sendt. & Quineira & arv & zoo & 21468 \\
\hline Solanum inaequale Vell. & Quina-amarela & arv & zoo & 21535 \\
\hline Solanum sp. 1 & - & arv & zoo & 21621 \\
\hline Solanum sp. 2 & - & arb & zoo & 21756 \\
\hline Solanaceae 1 & - & arb & zoo & 35965 \\
\hline Solanaceae 2 & - & lian & zoo & 35551 \\
\hline Solanaceae 3 & - & arb & zoo & 35829 \\
\hline Solanaceae 4 & - & herb & - & 35960 \\
\hline Solanaceae 5 & - & arv & zoo & 35951 \\
\hline Solanaceae 6 & - & arv & zoo & 35832 \\
\hline \multicolumn{5}{|l|}{ Symplocaceae } \\
\hline Symplocos cf. celastrinea Mart. ex Miq. & - & arv & zoo & - \\
\hline Symplocos cf. tetrandra Mart. & - & arv & zoo & 21815 \\
\hline Symplocos uniflora (Pohl.) Benth. & - & arv & zoo & 21619 \\
\hline \multicolumn{5}{|l|}{ Urticaceae } \\
\hline Cecropia glaziovi Snethlage & Embaúba & arv & zoo & - \\
\hline Cecropia pachystachya Trécul & Embaúba & arv & zoo & 21780 \\
\hline Coussapoa microcarpa (Schott) Rizzini & Figueirinha & est & zoo & 21763 \\
\hline Pilea rhizobola Miq. & - & herb & - & 35955 \\
\hline Pilea sp. & - & herb & - & 36012 \\
\hline Pourouma guianensis Aubl. & Mandioqueira & arv & zoo & 21371 \\
\hline Urera baccifera (L.) Gaudich. ex Wedd. & Urtiga & arb & zoo & 36311 \\
\hline Urera sp. & - & arv & zoo & 35900 \\
\hline \multicolumn{5}{|l|}{ Verbenaceae } \\
\hline Aegiphylla sellowiana Cham. & Cajuja & arv & zoo & 21578 \\
\hline Citharexylum myrianthum Cham. & Tucaneiro & arv & zoo & 21536 \\
\hline Vitex cf. sellowiana Cham. & - & arv & zoo & - \\
\hline Vitex sp. & Tarumã & arv & zoo & 27801 \\
\hline \multicolumn{5}{|l|}{ Viscaceae } \\
\hline Phoradendron sp. & Enxerto & par & zoo & 36431 \\
\hline Viscaceae & - & par & zoo & 25281 \\
\hline \multicolumn{5}{|l|}{ Vitaceae } \\
\hline Cissus cf. campestris (Baker) Planch. & - & lian & zoo & 35584 \\
\hline \multicolumn{5}{|l|}{ Vochysiaceae } \\
\hline Callisthene cf. dryadum A.P. Duarte & - & arv & anemo & 21792 \\
\hline Vochysia cf. bifalcata Warm. & Guaricica & arv & anemo & - \\
\hline
\end{tabular}


Tabela 1. Continuação

\begin{tabular}{|c|c|c|c|c|}
\hline Família / Espécie & Nome comum & Hábito & Dispersão & HRCB \\
\hline \multicolumn{5}{|l|}{ Indeterminada } \\
\hline Indeterminada & - & arv & - & 2776 \\
\hline
\end{tabular}

Araceae

Anthurium cf. rudgeanum Schott

$\begin{array}{llll}- & \text { epf } & \text { zoo } & 21364 \\ - & \text { epf } & \text { zoo } & 27797 \\ - & \text { epf } & \text { zoo } & 27783 \\ - & \text { epf } & \text { zoo } & 27795 \\ - & \text { epf } & \text { zoo } & 27757 \\ \text { - } & \text { epf } & \text { zoo } & 27784 \\ \text { - } & \text { epf } & \text { zoo } & 27792 \\ \text { - } & \text { epf } & \text { zoo } & 27752 \\ \text { - } & \text { epf } & \text { zoo } & 21362 \\ \text { - } & \text { herb } & \text { zoo } & 35565 \\ \text { Timbó-peva } & \text { epf } & \text { zoo } & 21848 \\ \text { Banana-de-macaco } & \text { epf } & \text { zoo } & 27785 \\ \text { - } & \text { epf } & \text { zoo } & 27780 \\ \text { Tucuá } & \text { epf } & \text { zoo } & 27763 \\ \text { Imbé } & \text { epf } & \text { zoo } & 27794 \\ \text { - } & \text { epf } & \text { zoo } & 27786 \\ \text { - } & \text { epf } & \text { zoo } & 27793 \\ \text { - } & \text { epf } & \text { zoo } & 27779 \\ \text { - } & \text { zoo } & 27777 \\ \text { - } & \text { zoo } & 27825\end{array}$

Syngonium podophyllum Schott

Arecaceae

Astrocaryum aculeatissimum (Schott) Burret

Bactris setosa Mart.

Brejaúva

Tucum

Bactris vulgaris Barb.Rodr.

Euterpe edulis Mart.

Palmiteiro

Geonoma elegans Mart.

Guaricana

Guaricana

Geonoma gamiova Barb.Rodr.

Guaricana

arv

$\operatorname{arv}$

ZOO

Geonoma schottiana Mart.

arb

ZOO

arv

zOO

Bromeliaceae

Billbergia amoena (Lodd.) Lindley

Nidularium billbergioides (Schultes f.) L.B. Sm.

Nidularium innocentii var. paxianum (Mez.) L.B. Sm.

Tillandsia tenuifolia L.

arb

ZOO

arb

zOO

Vriesea carinata Wawra

$\begin{array}{ll}\text { epf } & \text { zoo } \\ \text { epf } & \text { zoo } \\ \text { epf } & \text { zoo } \\ \text { epf } & \text { anemo } \\ \text { epf } & \text { anemo }\end{array}$


Tabela 1. Continuação

\begin{tabular}{|c|c|c|c|c|}
\hline Família / Espécie & Nome comum & Hábito & Dispersão & HRCB \\
\hline \multicolumn{5}{|l|}{ Bromeliaceae (cont.) } \\
\hline Vriesea drepanocarpa (Baker) Mez. & - & epf & anemo & 36270 \\
\hline Vriesea ensiformis (Vell.) Beer & Bromélia & epf & anemo & 25285 \\
\hline Vriesea incurvata Gaudch. & - & epf & anemo & 36272 \\
\hline Vriesea rodigasiana E. Morren & - & epf & anemo & 36264 \\
\hline \multicolumn{5}{|l|}{ Commelinaceae } \\
\hline Dychorisandra thyrsiflora J.G. Mikan & - & herb & zoo & 21872 \\
\hline Dychorisandra sp. 1 & - & arb & zoo & 25427 \\
\hline Dychorisandra sp. 2 & - & arb & $\mathrm{zOO}$ & 25429 \\
\hline \multicolumn{5}{|l|}{ Costaceae } \\
\hline Costus spiralis Rosc. & - & arb & zoo & 21871 \\
\hline \multicolumn{5}{|l|}{ Cyclanthaceae } \\
\hline Asplundia polymera (Hand.-Mazz.) Harling & - & epf & zoo & 25529 \\
\hline \multicolumn{5}{|l|}{ Сyperaceae } \\
\hline Hypolytrum schraderianum Nees & - & herb & - & 35945 \\
\hline Pleurostachys gaudichaudii Brongn. & - & herb & anemo & 25431 \\
\hline Pleurostachys urvilleii Brongn. & - & herb & anemo & 32309 \\
\hline Scleria sp. & - & herb & - & 36276 \\
\hline \multicolumn{5}{|l|}{ Dioscoreaceae } \\
\hline Dioscorea sp. & - & lian & - & 36278 \\
\hline \multicolumn{5}{|l|}{ Heliconiaceae } \\
\hline Heliconia spathocircinata Aristeg. & Caeté-banana & herb & zoo & 21539 \\
\hline Heliconia velloziana L. Emygd. & Caeté-banana & herb & zoo & 21646 \\
\hline \multicolumn{5}{|l|}{ Iridaceae } \\
\hline Neomarica sp. & - & herb & auto & 35863 \\
\hline \multicolumn{5}{|l|}{ Marantaceae } \\
\hline Calathea aff. longifolia Klotzsch & Caeté & herb & zoo & 21833 \\
\hline Calathea communis Wanderley \& Vieira & Caeté & herb & zoo & 35845 \\
\hline Calathea cylindrica (Roscoe) K. Schum. & - & herb & zoo & - \\
\hline Ctenanthe cf. kummeriana Eichl. & - & herb & zoo & - \\
\hline Stromanthe thalia (Vell.) J.M.A.Braga & - & herb & zoo & 35573 \\
\hline Stromanthe tonckat Eichl. & - & herb & $\mathrm{zOO}$ & 25023 \\
\hline \multicolumn{5}{|l|}{ Musaceae } \\
\hline Musa velutina $\mathrm{H}$. Wendl. \& Drude & Bananinha & arb & zoo & 21837 \\
\hline \multicolumn{5}{|l|}{ Orchidaceae } \\
\hline Aspidogyne fimbrillaris (B.S. Wms.) Garay & - & herb & anemo & 35939 \\
\hline Barbosella gardneri (Lindl.) Schltr. & - & epf & anemo & 36290 \\
\hline Dichaea pendula Cogn. & - & epf & anemo & 32310 \\
\hline
\end{tabular}


Tabela 1. Continuação

\begin{tabular}{|c|c|c|c|c|}
\hline Família / Espécie & Nome comum & Hábito & Dispersão & HRCB \\
\hline \multicolumn{5}{|l|}{ Orchidaceae (cont.) } \\
\hline Epidendrum ramosum Jacq. & - & epf & anemo & 36291 \\
\hline Epidendrum rigidum Jacq. & - & epf & anemo & 36294 \\
\hline Epidendrum strobiliferum Rchb. f. & - & epf & anemo & 36299 \\
\hline Erythrodes arietina (Rchb. f. \& Warm.) Ames & - & herb & anemo & 35944 \\
\hline Maxillaria ochroleuca Lodd. ex Lindl. & - & epf & anemo & 36296 \\
\hline Octomeria grandiflora Lindl. & - & epf & anemo & 36292 \\
\hline Pelexia cf. macropoda (Barb. Rodr.) Schltr. & - & herb & anemo & 35940 \\
\hline Prosthechea triptera (Hook.) W.E. Higgins & - & epf & anemo & 36293 \\
\hline Scaphyglottis brasiliensis (Schltr.) Dressler & - & epf & anemo & 36298 \\
\hline Scaphyglottis modesta (Rchb. f) Schltr. & - & epf & anemo & 36295 \\
\hline Orchidaceae & - & herb & anemo & 35937 \\
\hline \multicolumn{5}{|l|}{ Poaceae } \\
\hline Olyra sp. 1 & - & herb & zoo & - \\
\hline Olyra sp. 2 & - & herb & zoo & - \\
\hline \multicolumn{5}{|l|}{ Smilacaceae } \\
\hline Smilax sp. & Dente-de-cachorro & lian & zoo & 21448 \\
\hline \multicolumn{5}{|l|}{ Zingiberaceae } \\
\hline Renealmia sp. & Capitiu & arb & zoo & 27772 \\
\hline
\end{tabular}

* icluindo os grupos parafiléticos

** Durante o levantamento não foram observados indivíduos de Ficus com hábito estrangulador. Por isso, as cinco espécies foram consideradas como arbóreas, mesmo que, eventualmente, algumas delas possam apresentar hábito estrangulador. 
Tabela 2. Número de espécies distribuídas pelas formas de vida na Floresta Atlântica da Base Saibadela, no Parque Estadual Intervales, Sete Barras-SP.

\begin{tabular}{lcccc}
\hline Forma de vida & eudicotiledôneas * & monocotiledôneas & Total & $\%$ \\
\hline árvore & 246 & 3 & 249 & $(57,1)$ \\
arbusto & 31 & 9 & 40 & $(9,2)$ \\
herbácea & 20 & 21 & 41 & $(9,4)$ \\
liana & 43 & 2 & 45 & $(10,3)$ \\
estranguladora & 3 & - & 3 & $(0,7)$ \\
epífita & 16 & 39 & 55 & $(12,6)$ \\
parasita & 3 & - & 3 & $(0,7)$ \\
\hline
\end{tabular}

* inclui os grupos parafiléticos

Tabela 3. Forma de vida e modos de dispersão de espécies da Floresta Atlântica na Base Saibadela, Parque Estadual Intervales, Sete Barras, SP.

\begin{tabular}{lcccc}
\hline Forma de vida & Zoocoria & Anemocoria & Hidrocoria & Autocoria \\
\hline árvore & 217 & 23 & - & 6 \\
arbusto & 37 & - & - & 2 \\
herbácea & 20 & 7 & 2 & 3 \\
liana & 24 & 14 & - & 3 \\
estranguladora & 2 & 1 & - & - \\
epífita & 35 & 20 & - & - \\
parasita & 2 & - & - & - \\
\hline Total & $338(80,7 \%)$ & $65(15,5 \%)$ & $2(0,5 \%)$ & $14(3,3 \%)$ \\
\hline
\end{tabular}

